\title{
Influence of a 12-week physical activity program on leptin resistance in metabolic syndrome: a preliminary study
}

\author{
Parameshwar Anche $^{1} \cdot$ G. Arun Maiya ${ }^{1} \cdot$ Shobha Ullas Kamath ${ }^{2} \cdot$ B. Ananthakrishna Shastry ${ }^{3}$
}

Received: 22 June 2020 / Accepted: 20 January 2021 / Published online: 9 March 2021

(C) The Author(s) 2021

\begin{abstract}
Purpose Metabolic syndrome is a condition with clustering of risk factors like insulin resistance, obesity, dyslipidemia, and hypertension. Leptin is a protein of obese gene produced by white adipose tissue. Leptin resistance is the insensitivity of leptin in hypothalamus despite high amounts in blood causing obesity and metabolic syndrome. The study focused on the influence of a 12-week physical activity promotion program on leptin resistance in people with metabolic syndrome

Methods After approval from institutional ethics committee (IEC 343-2018), 18 participants (males $N=4$, females $N=14$ ) of age group $45.0 \pm 7.6$ years with metabolic syndrome according to (NCEP ATP-III) criteria were included in the study. The participants underwent a 12-week physical activity program consisting of $150 \mathrm{~min}$ of moderate to vigorous activity per week as per GPAQ domains - work, transport, and recreation. The outcomes were measured at baseline and after 12 weeks.

Results Out of 18 participants, 10 participants who completed the study were analysed. Twelve-week physical activity showed significant changes in waist circumference $(p=0.047)$, post prandial blood glucose $(p=0.0396)$, triglycerides $(p=0.0323)$, body mass index $(p=0.0056)$, subcutaneous fat $(p=0.0354)$, and basal metabolic rate $(p=0.0035)$. Fasting blood glucose $(p=0.254)$, lipid profiles (total cholesterol $(p=0.062)$ ), high-density lipoprotein $(p=0.367)$, low-density lipoprotein $(p=0.641)$, and leptin showed insignificant change $(p=0.328)$. Global physical activity questionnaire showed significant change $(p=0.0254)$ suggesting changes in physical activity behaviors.

Conclusion From present study, it is concluded that a 12-week physical activity promotion program brought marginal changes in leptin levels and has potential to modify metabolic syndrome parameters and improve physical activity.
\end{abstract}

Keywords Metabolic syndrome $\cdot$ Leptin resistance $\cdot$ Physical activity $\cdot$ Body composition

G. Arun Maiya

arun.maiya@manipal.edu; ajmaiya@gmail.com

Parameshwar Anche

param.anche@gmail.com

Shobha Ullas Kamath

shobha.kamath@manipal.edu

B. Ananthakrishna Shastry

shastryba@yahoo.com

1 Department of Physiotherapy, Centre for Diabetic Foot Care \& Research, Manipal College of Health Professions, Manipal Academy of Higher Education, Manipal, Karnataka 576104, India

2 Department of Biochemistry, Kasturba Medical College, Manipal Academy of Higher Education, Manipal, India

3 Department of Medicine, Kasturba Medical College, Manipal Academy of Higher Education, Manipal, India

\section{Introduction}

Metabolic syndrome (MetS) is a condition characterized by clustering of several known risk factors like insulin resistance (IR), obesity, atherogenic dyslipidemia, and hypertension (HTN) [1]. It was named as syndrome $\mathrm{X}$ by Gerald Raven in 1988 with aggregation of several important cardiovascular risk factors. Visceral fat is considered as a major risk factor for coronary heart disease and type 2 diabetes mellitus [2]. In addition to this, elevated serum levels of fibrinogen and tissue plasminogen activator inhibitor (PAI) are related to metabolic factors of CHD. Low-density lipoprotein (LDL) is associated with insulin resistance, obesity, and type 2 diabetes. Dyslipidemia is also considered as one of the risk factors of MetS with increased serum triglycerides, decreased highdensity lipoprotein (HDL), and increased LDL. Other contributing conditions of MetS include endothelial dysfunction [3], inflammation, and oxidative stress [4]. 
Leptin is a 167 -amino acid protein, a product of obese gene produced by white adipose tissue. It functions as endocrine organ with numerous functions apart from fat storage [5]. It works on hypothalamus and acts to decrease food intake and increase metabolism. It helps in decreasing appetite, increasing energy expenditure, and regulating glucose homeostasis [6]. Congenital leptin deficiency has shown to cause severe obesity, glucose intolerance, and insulin resistance in humans. Increased serum leptin can be due to increased FFA, insulin stimulation, oestrogen, TNF $\alpha$, and impaired renal clearance. Increased leptin levels are associated with metabolic syndrome [7]. High levels of leptin are seen in obese individuals due to defect in leptin receptor, inhibition in leptin signalling, and alterations in leptin transport in blood brain barrier [8]. Leptin resistance occurs in $\beta$ cells which may lead to hyperinsulinemia which in turn causes less energy expenditure and leads to metabolic syndrome. There are no specific criteria to diagnose leptin resistance. Studies showed that hyperleptinemia is an important marker for leptin resistance $[9,10]$. Leptin resistance could be due to reduction in expression of short and long isoforms of leptin receptors (OBRa \& $\mathrm{OBRb}$ ) in hypothalamus, hepatocytes, adipose tissue, and muscles [11,12].

Strong evidence exists that both children and adults benefit from being physically active. Benefits of physical activity include lower risk of chronic diseases such as obesity, heart disease, diabetes, cancer, and depression [13-19]. It is widely accepted that physical activity is a low-cost alternative to disease treatment and prevention [20]. Phillip Tuso discussed the five strategies to be physically active which are to (1) measure physical activity as a vital sign; (2) encourage patients to be physically active at least 150 min per week; (3) create healthy environments by making it easier for patients to be physically active where they live, learn, work, play, and pray; (4) monitor disease incidence of patients who are physically active vs those who are not physically active; and (5) spread best practices [20]. MetS affects people whose excess body weight, and sedentary lifestyle determine the phenotype expression of genetic trait [21]. Maintaining normal weight and practising physical activity remain the most effective prevention strategy of MetS [22]. Management of MetS should be based on effective weight loss program and exercise program which needs specific strategies for the maintenance of the program. Weight loss is associated with significant improvements in clinical components of MetS, and there is association between moderate weight loss and improvements in metabolic profile [23]. Physical activity (PA) has a major role in the treatment of MetS. Cardiopulmonary fitness has shown to modify cardiovascular events, thus providing a strong and protective effect on cardiovascular health. Several studies have shown that increased levels of physical activity are associated with less prevalence and incidence of MetS [24].
However, the effect of PA promotion on leptin resistance among people with metabolic syndrome is not known. The purpose of this study is to determine the influence of a 12week PA promotion program on leptin resistance in people with metabolic syndrome (Fig. 1).

\section{Methods}

After approval from institutional ethics committee (IEC 3432018), 18 participants (males $N=4$, females $N=14$ ) with age group $45.0 \pm 7.6$ with 3 out of 5 criteria of metabolic syndrome according to the National Cholesterol Education Program Adult Treatment Protocol III (NCEP ATP-III) criteria were included in the study (Fig. 2). In the present study, the screened participants have been advised to continue the medication as per the physician advice. Standard diet prescription was given by the hospital dietician. Participants were excluded if they have a history of smoking past 6 months, cardiovascular disease, stroke, transient ischemic attack (TIA), any cognitive impairment, any systemic illness, and if they are having any other contraindications for PA as mentioned in PARQ+ physical activity readiness questionnaire.

\section{Study design}

The study is preliminary pre-post non-randomized interventional with a single group of participants with metabolic syndrome. The sample size for this study was estimated as general rule of thumb as suggested by Julious [25]. The participants were selected using convenience sampling from the Kasturba Hospital after a thorough medical evaluation and clearance for physical activity from the physician.

\section{Procedure}

The participants were enrolled into the study after physician clearance and low risk as per AHA/ACSM pre-participation questionnaire and PARQ+ physical activity readiness questionnaire. If the participants were found to be under moderate or severe risk category, they were advised to consult a physician before the start of any physical activity. Participants who were included in the study were assessed for fasting blood sugar (FBS), waist circumference (WC), blood pressure (BP), body composition, physical activity, leptin, lipid profile, and basal metabolic rate (BMR). BP is taken with digital sphygmomanometer Omron HEM-7113 (Omron Health Care Inc, Illinois) (Accuracy: Pressure: $\pm 3 \mathrm{mmHg}$ Pulse: $\pm 5 \%$ of display reading) after making the participant rest for $3 \mathrm{~min}$ in a quiet room. 


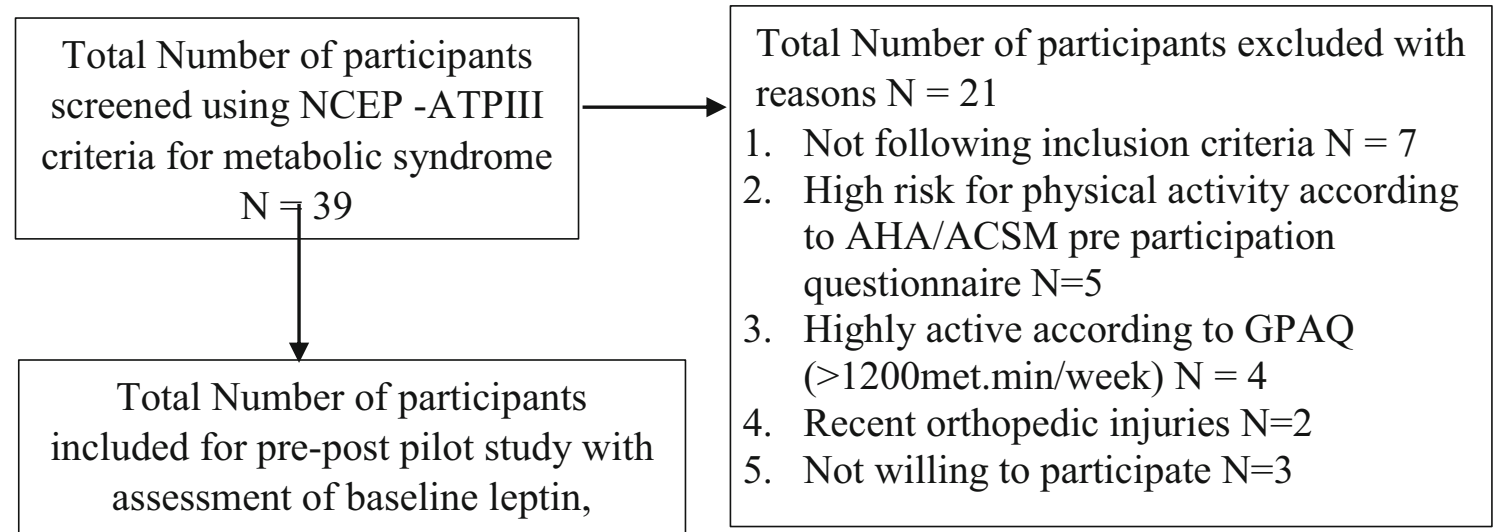

\section{Dropouts from the study} with reasons

1. Lost to follow up $=2$

2. Incomplete intervention $=4$

3. Could not find time for intervention $=2$
Fig. 1 Flow chart of the participant recruitment and analysis

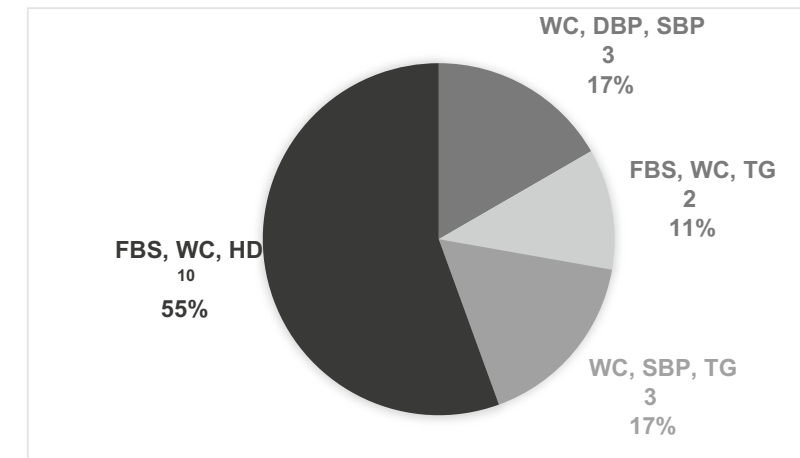

Fig. 2 Diagnostic criteria of metabolic syndrome according to NCEPATP III

\section{Total Number of} participants included for analysis of post

intervention outcomes leptin, GPAQ, metabolic syndrome parameters and body composition $\mathrm{N}=10$

$$
\mathrm{N}=10
$$

\section{Body composition and anthropometry}

Body composition (BC) was analysed using Omron Karada Scan HBF 362 bio impedance analyser (BIA) (Omron Health Care, Inc, Illinois). Body composition included total body fat (TBF), subcutaneous fat (SCF), visceral fat (VF), musculoskeletal mass (MSM), and basal metabolic rate (BMR). For assessing BC using BIA, the participant was instructed to wear light clothing, 8-h fasting, avoiding of caffeine intake, alcohol intake and exercise $2 \mathrm{~h}$ prior to test, and no fluid intake $2 \mathrm{~h}$ prior to the test. The participant age, gender, and height are entered into the analyser, and the participant is made to stand on the BIA platform. TBF, SCF, VF, MSM, and BMR readings are recorded. $\mathrm{WC}$ is taken in centimetres with a non- 
stretching tape at the level above iliac crest. Basal metabolic rate was calculated using Mifflin-St Jeor equation

$$
\begin{aligned}
\operatorname{BMR}(\mathrm{kcal} / \text { day })= & 10 * \text { weight }(\mathrm{kg})+6.25 * \text { height }(\mathrm{cm}) \\
& -5 * \text { age }(\mathrm{y})+\mathrm{s}(\mathrm{kcal} / \text { day }),
\end{aligned}
$$

where $s$ is +5 for males and -161 for females.

\section{Biochemical measurements}

Blood sample was taken after $8 \mathrm{~h}$ of fasting state to measure FBS, total cholesterol (TC), triglycerides (TG), high-density lipoproteins (HDL), low-density lipoprotein (LDL), and leptin. FBS was analysed using hexokinase method, TC was analysed using CE-CHOD-POD method (Atlas Medicals, Cambridge), triglycerides were analysed using GPO trinder method (BQ kits Inc, San Francisco, CA), HDL was assessed using direct-homogenous assay, and LDL was calculated using the Friedewald formula. Leptin was analysed by enzyme-linked immunosorbent assay (ELISA) sandwich technique using commercially available kits DBC ELISA (Diagnostic Biochem Canada Inc) with specificity of 0.50 $\mathrm{ng} / \mathrm{ml}$, intra assay CV $3.7 \%$, and interassay CV of $5.9 \%$.

\section{Physical activity measurement}

PA was measured using the World Health Organization global physical activity questionnaire (WHO-GPAQ). It contains 4 domains: work, transport, recreational, and sedentary time. GPAQ demonstrated good-to-very good test-retest reliability with time intervals that ranged from 3 days to 2 weeks [21]. GPAQ is used to assess the PA for a week and is expressed in met.min/week. The individual is said to be sedentary if the GPAQ score is less than 600 met.min/week.

\section{PA promotion program}

After assessing the PA using GPAQ, the participants were given a 12-week PA program which consisted of $150 \mathrm{~min} /$ week moderate to vigorous PA. Before giving the PA, the participants were asked about the daily routine and explained how they can modify their work routine into PA by monitoring themselves using commercially available fitness trackers which measure the step count. The participants using these fitness trackers were instructed to maintain 10,000 steps/day. People who did not have access to fitness trackers were given PA program according to the WHO compendium of physical activities which contain list of activities with specific MET values. To maintain adherence, the participants were advised to do PA in their convenient time without disturbing their daily routine but were instructed to maintain 150 min moderate to vigorous PA per week. The PA included various activities like brisk walking, gardening, house chores, workplace physical activity, and leisure time physical activities like group activities in a park which included yoga, general drills, and light jogging. To monitor PA, the participants were called by telephone every week to enquire about the activities they are doing. The progression of PA was measured every third week using GPAQ. The target was to achieve 150 min of physical activity per week. All the participants had less than $60 \mathrm{~min} /$ week of physical activity at the start of the study. The target was to increase the physical activity in a steady phase until they reach the required $150 \mathrm{~min} /$ week target. After 12 weeks, the participants are re-assessed for the outcomes like leptin, GPAQ, BP, TC, TG, HDL, LDL, BMR, SCF, VF, MSM, and WC.

\section{Statistical analysis}

Descriptive statistics were presented as mean and standard deviation. Shapiro-Wilk test was performed for normal distribution. Paired $t$ test was performed to analyse the difference preand post-intervention. $p$ value was set at $p<0.05$ at $95 \%$ CI.

\section{Results}

Participants $(N=18)$ aged $30-50$ years of either gender who were diagnosed with metabolic syndrome under NCEP ATP

Table 1 Baseline characteristics of the participants

Baseline characteristics of the participants $N=18$

\begin{tabular}{ll}
\hline Variable & Mean \pm SD \\
\hline Age (years) & $47.2 \pm 4.86$ \\
GPAQ (met.min/wk) & $231.7 \pm 138.18$ \\
LEPTIN (ng/ml) & $25.9 \pm 10.69$ \\
Metabolic syndrome parameters & Mean \pm SD \\
WC (cm) & $101.8 \pm 8.5$ \\
SBP (mmhg) & $134.2 \pm 3.96$ \\
DBP (mmhg) & $88.2 \pm 4.62$ \\
FBS (mg/dl) & $152.5 \pm 42.44$ \\
HDL (mg/dl) & $43.3 \pm 10.14$ \\
LDL (mg/dl) & $129.1 \pm 32.7$ \\
TG (mg/dl) & $177.1 \pm 34.81$ \\
BMI (kg/m $\left.{ }^{2}\right)$ & $28.3 \pm 4.57$ \\
PPBS (mg/dl) & $188.8 \pm 44.39$ \\
Body composition & Mean \pm SD \\
TC (mg/dl) & $214.1 \pm 29.24$ \\
SCF (\%) & $28.9 \pm 3.7$ \\
VF(\%) & $11.2 \pm 1.6$ \\
MSM(\%) & $23.4 \pm 5.8$ \\
BMR (Kcal) & $2025.54 \pm 138.6$ \\
TF (\%) & $31.3 \pm 8.5$ \\
\hline
\end{tabular}


III criteria were recruited for the study. Ten participants who completed the study were included in the analysis. The main parameters of the MetS are presented in Table 1 which included all 18 participants. All the participants had increased waist circumference, dyslipidemia, hyperglycemia, and elevated blood pressure according to NCEP ATP III for metabolic syndrome (Fig. 3) . Table 2 describes the changes in all outcomes with a 12-week physical activity of the 10 participants who completed the study. Two participants who were enrolled into the study were not available for the post-intervention analysis. One participant was available but followed intervention less than 6 weeks so could not be analysed; 3 participants had adherence issues due to their work-related stress and stopped intervention abruptly. Two participants who did not find time for the intervention described of unable to balance time due to change of their job. The results showed that the mean physical activity levels of the participants were $330.4 \pm 85.6 \mathrm{met} . \mathrm{min} /$ week which is less than the recommended levels of $>600 \mathrm{met}$.min/week according to GPAQ. This clearly had shown the elevation of MetS parameters showing the importance of physical activity. All the participants were diagnosed as leptin resistant with elevated leptin levels $(25.5 \pm 9.3$ $\mathrm{ng} / \mathrm{ml})$ which correlated with the elevation of BMI (27.5 \pm 2.36 $\mathrm{kg} / \mathrm{m}^{2}$ ). We also observed that all the participants had elevated BMR levels (1954.35 $\pm 142.5 \mathrm{kcal})$ suggesting decreased lean mass and increased total fat mass (30.7 \pm 6.8$)$. The 12-week physical activity program brought changes in some parameters of MetS. The participants were motivated and explained the benefits of physical activity which brought a significant change in physical activity levels $(p=0.0254)$. As the physical activity levels increased, WC changed significantly $(p=0.047)$. PPBS has shown significant change $(p=0.0396)$, whereas FBS did not show significant change $(p=0.254)$. No significant changes were seen in lipid profiles (TC ( $p=0.062)$, HDL $(p=0.367)$, LDL $(p=0.641)$ ). But triglycerides have shown significant change in 12 weeks $(p=0.0323)$. Body composition assessment has shown significant changes in BMI $(p=0.0056)$, SCF $(p=0.0354)$, and $\operatorname{BMR}(p=0.0035)$. Leptin levels did not show significant change $(p=0.328)$ despite changes in BMI and SF. Out of 10 participants who were analysed, 1participant had improvement in three

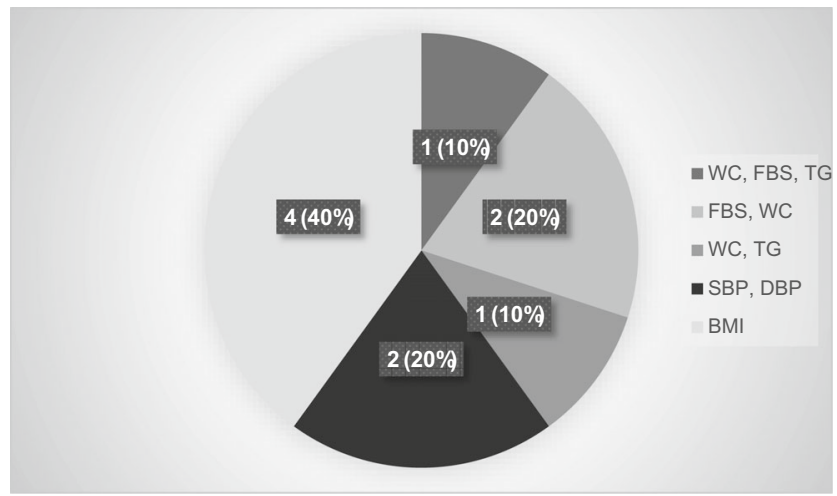

Fig. 3 Changes in individual parameters of metabolic syndrome according to NCEP-ATP III
Table 2 Mean change in leptin, body composition, and metabolic syndrome parameters of the participants who completed the study

\begin{tabular}{|c|c|c|c|}
\hline Variable & Baseline $(N=10)$ & 12 weeks $(N=10)$ & $p$ value \\
\hline Leptin (ng/ml) & $25.5 \pm 9.3$ & $23.7 \pm 11.5$ & \\
\hline GPAQ (met.min & /wk) $\quad 330.4 \pm 85.6$ & $420.5 \pm 122.4$ & $0.0254 *$ \\
\hline \multicolumn{4}{|c|}{ Metabolic syndrome parameters } \\
\hline $\mathrm{WC}(\mathrm{cm})$ & $98.6 \pm 5.4$ & $96.4 \pm 2.8$ & $0.047 *$ \\
\hline SBP (mmhg) & $132.5 \pm 4.25$ & $131.5 \pm 3.68$ & 0.549 \\
\hline DBP (mmhg) & $86.2 \pm 5.83$ & $86.8 \pm 2.95$ & 0.269 \\
\hline FBS (mg/dl) & $148.5 \pm 39.65$ & $147.7 \pm 38.63$ & 0.254 \\
\hline PPBS (mg/dl) & $185 \pm 41.36$ & $183.54 \pm 36.52$ & $0.0396^{*}$ \\
\hline \multicolumn{4}{|l|}{ Lipid profile } \\
\hline $\mathrm{TC}(\mathrm{mg} / \mathrm{dl})$ & $213.4 \pm 29.24$ & $212.2 \pm 25.63$ & 0.062 \\
\hline HDL (mg/dl) & $45.3 \pm 9.6$ & $43.9 \pm 3.8$ & 0.367 \\
\hline LDL (mg/dl) & $132.4 \pm 27.5$ & $130.7 \pm 26.8$ & 0.641 \\
\hline $\mathrm{TG}(\mathrm{mg} / \mathrm{dl})$ & $180.6 \pm 26.3$ & $176.3 \pm 26.7$ & $0.0323 *$ \\
\hline \multicolumn{4}{|c|}{ Body composition } \\
\hline BMI $\left(\mathrm{kg} / \mathrm{m}^{2}\right)$ & $27.5 \pm 2.36$ & $25.3 \pm 2.89$ & $0.0056^{*}$ \\
\hline $\mathrm{SCF}(\%)$ & $29.3 \pm 5.6$ & $26.5 \pm 2.92$ & $0.0354 *$ \\
\hline $\operatorname{VF}(\%)$ & $10.8 \pm 0.8$ & $9.7 \pm 1.6$ & 0.296 \\
\hline $\operatorname{MSM}(\%)$ & $23.5 \pm 4.6$ & $22.8 \pm 4.9$ & 0.074 \\
\hline BMR (Kcal) & $1954.35 \pm 142.5$ & $2026.4 \pm 142.9$ & $0.0035^{*}$ \\
\hline $\mathrm{TF}(\%)$ & $30.7 \pm 6.8$ & $28.9 \pm 5.6$ & 0.086 \\
\hline
\end{tabular}

$* p$ value $<0.05$

criteria of metabolic syndrome (WC, FBS, and TG), 2 participants had improvements in 2 criteria of Mets (FBS and WC), 1 participant showed improvement in parameters (WC, TG), 2 participants showed improvement in SBP and DBP, and 4 participants showed changes in BMI (Fig. 2).

\section{Discussion}

The present study aimed to find the effect of a 12-week physical activity promotion on leptin resistance in people with metabolic syndrome. This preliminary study results showed changes in some parameters of metabolic syndrome, body composition, and changes in PA behavior. Out of 18 participants, 10 participants completed the study (Fig. 1). The reason could be that unlike exercise, PA is more generalized and does not follow a structured protocol, and we observed that participants were not aware of the difference between an exercise protocol and physical activity. Moreover, awareness and selfmotivation play an important role in maintaining the adherence to program. Previous studies were in line with our findings about the adherence to physical activity [26]. In our study, the dropped-out participants reported that they did not have time for exercise mostly due to their work schedule and type of work. Participants who enrolled into the study were 
clearly explained the importance of physical activity to maintain optimum health and bring changes in metabolic syndrome parameters and lead a healthy lifestyle. Participants were encouraged to use technology like smart wearables and smart phone apps which monitor and provide motivation to increase physical activity and continue the new active lifestyle. There is a strong evidence on benefits of technology in improving physical activity [27-31]. Overall, the 12-week PA promotion program has brought changes in most metabolic syndrome parameters. Leptin levels did not change significantly despite significant decrease in BMI levels. Previous studies done by Xenachis $\mathrm{C}$ et al. [32] found that a 7\% decrease in body weight was associated with a $22 \%$ reduction in serum leptin; Bastard et al. [33] found that decrease in leptin with a decrease of $2.1 \mathrm{~kg} / \mathrm{m}^{2}$ in BMI was followed by a $45 \%$ reduction in leptin levels. The discrepancy in leptin levels in our study may be due to sample size being too low to determine the effect of this intervention in decreasing leptin resistance. Leptin levels remained stable despite decrease in SCF. The participants were mostly females, and they have comparatively higher fat content than male population. This could be the reason of unchanged leptin levels. The changes in BMR can be attributed to change in SF and non-significant increase in MSM. Because BMR is the marker of lean body mass, increase in MSM and decrease in SF could decrease in BMR suggesting healthy weight loss. We have observed a statistically insignificant change in VF. The reason could be the unique characteristics of VF like increased blood flow and more response to norepinephrine. Studies have shown that increase in VF could lead to increased BMR [34]. In our study, MSM and VF have shown insignificant changes supporting the statement of higher BMR values. Further assessment is needed to assess the difference in BMR per unit decrease in VF and per unit increase in MSM. SBP and DBP did not show a significant change suggesting that the intervention could not bring out much change in sympathetic system which regulates the blood pressure. The results for SBP and DBP were inconsistent with other studies [35-37] probably due to small sample size. Although there was insignificant change in TC, TG had shown a significant change post-intervention suggesting that a moderate amount of physical activity for longer duration could help in fat metabolism especially TG which is source of energy for long duration steady-state activity. E.G. Oh et al. [38] found a decrease in TG with a 3month total lifestyle modification which supported our results concerning decrease in TG. In the present study, based on the WHO-GPAQ questionnaire, all the participants were sedentary with the MET value $<600 \mathrm{met} . \mathrm{min} /$ week. Our main purpose of the study was to sensitize people about the physical activity and to include physical activity in their day-to-day life. Based on our results, we observed that all participants' physical activity has been improved. However, as a part of ongoing research, we had followed them telephonically regarding the continuation physical activity promotion, but the data was not reported in the present study.
The strengths of the study were that the 12-week physical activity promotion program could bring changes in metabolic syndrome parameters, decrease sedentary time, and improve physical activity. Waist circumference showed a significant change with the intervention which suggests that healthy lifestyle with physical activity as a daily routine could bring out weight loss and help in improving optimum health.

The limitations of the study were small sample size and disparity between gender which could have interfered with the results. As this is a longitudinal study and no control group was involved, there was no comparison for the results. Another limitation of the study was high attrition rate showing poor adherence to the program which needs to be addressed in the future studies. Future studies should investigate a randomized controlled trial with appropriate control group and address the above issues for more clear results and the effect of intervention.

Future studies should also investigate association between leptin resistance and BMR, total fat, and effect of the PA intervention on these variables. However, our study was able to show a considerable change in the metabolic syndrome parameters, some components of body composition, and anthropometry.

\section{Conclusion}

From the present study, we have concluded that a 12 -week physical activity promotion program brought marginal changes in the leptin levels and has a potential to modify the metabolic syndrome parameters and changes in body composition and improve physical activity behavior.

Acknowledgment World Diabetes Foundation (WDF 15-941) "Diabetic Foot Care: Stepping Ahead"

Centre for Diabetic Foot Care and Research.

The Author acknowledges Ms. Gagana Herle. PhD Scholar, Centre for diabetic foot care and Research, MAHE, Manipal for her contribution in biochemical analysis involved in the study.

\section{Declarations}

Conflict of interest The authors declare no competing interests.

Informed consent Informed consent has been obtained from all the participants prior to the inclusion into the study.

Open Access This article is licensed under a Creative Commons Attribution 4.0 International License, which permits use, sharing, adaptation, distribution and reproduction in any medium or format, as long as you give appropriate credit to the original author(s) and the source, provide a link to the Creative Commons licence, and indicate if changes were made. The images or other third party material in this article are included in the article's Creative Commons licence, unless indicated otherwise in a credit line to the material. If material is not included in the article's Creative Commons licence and your intended use is not permitted by statutory regulation or exceeds the permitted use, you will need to obtain permission directly from the copyright holder. To view a copy of this licence, visit http://creativecommons.org/licenses/by/4.0/. 


\section{References}

1. Ross R, Aru J, Freeman J, Hudson R, Janssen I. Abdominal adiposity, and insulin resistance in obese men. Am J Physiol Endocrinol Metab. 2002;282:E657-63.

2. Van Zwieten PA. Endothelial dysfunction in hypertension. A critical evaluation. Blood Press Suppl. 1997;2:67-70.

3. Van Guilder GP, Hoetzer GL, Greiner JJ, Stauffer BL, DeSouza CA. Influence of metabolic syndrome on biomarkers of oxidative stress and inflammation in obese adults. Obesity. 2006;14:212731.

4. Haffner SM, Valdez RA, Hazuda HP, Mitchell BD, Morales PA, Stern MP. Prospective analysis of the insulin-resistance syndrome (syndrome X). Diabetes. 1992;41:715-22.

5. Defronzo RA, Ferrannini E. Insulin resistance: a multifaceted syndrome responsible for NIDDM, obesity, hypertension, dyslipidemia and atherosclerotic cardiovascular disease. Diabetes Care. 1991;14:173-94.

6. Bjorntorp P. "Portal" adipose tissue as a generator of risk factors for cardiovascular disease and diabetes. Arteriosclerosis. 1990;10:4936.

7. Despres JP, Lemieux I. Abdominal obesity and metabolic syndrome. Nature. 2006;444:881-7.

8. Golbidi S, Laher I. Exercise induced adipokine changes and the metabolic syndrome. J Diabetes Res. 2014;2014:726861.

9. Posey KA, Clegg DJ, Printz RL, Byun J, Morton GJ, Vivekanandan-Giri A, et al. Hypothalamic proinflammatory lipid accumulation, inflammation, and insulin resistance in rats fed a high-fat diet. Am J Physiol Endocrinol Metab. 2009;296(5): E1003-12.

10. Zubiría MG, Vidal-Bravo J, Spinedi E, Giovambattista A. Relationship between impaired adipogenesis of retroperitoneal adipose tissue and hypertrophic obesity: role of endogenous glucocorticoid excess. J Cell Mol Med. 2014;18(8):1549-61.

11. Rehman Khan A, Awan FR. Leptin resistance: a possible interface between obesity and pulmonary-related disorders. Int J Endocrinol Metab. 2016;14(1):e32586.

12. Schaab M, Kratzsch J. The soluble leptin receptor. Best Pract Res Clin Endocrinol Metab. 2015;29(5):661-70.

13. Groba C, Mayerl S, van Mullem AA, Visser TJ, Darras VM, Habenicht AJ, et al. Hypothyroidism compromises hypothalamic leptin signaling in mice. Mol Endocrinol. 2013;27(4):586-97.

14. Catenacci VA, Wyatt HR. The role of physical activity in producing and maintaining weight loss. Nat Clin Pract Endocrinol Metab. 2007;3(7):518-29.

15. Sattelmair J, Pertman J, Ding EL, Kohl HW 3rd, Haskell W, Lee IM. Dose response between physical activity and risk of coronary heart disease: a meta-analysis. Circulation. 2011;124(7):789-95.

16. Jeon CY, Lokken RP, Hu FB, van Dam RM. Physical activity of moderate intensity and risk of type 2 diabetes: a systematic review. Diabetes Care. 2007;30(3):744-52.

17. McTiernan A. Mechanisms linking physical activity with cancer. Nat Rev Cancer. 2008;8(3):205-11.

18. Martinsen EW. Physical activity in the prevention and treatment of anxiety and depression. Nord J Psychiatry. 2008;62(Suppl 47):259.

19. Harkin A. Muscling in on depression. N Engl J Med. 2014;371(24): 2333-4.

20. Tuso P. Strategies to increase physical activity. Perm J. 2015 Fall;19(4):84-8.

21. Dong M, Ren J. What fans the fire: insights into mechanisms of leptin in metabolic syndrome-associated heart diseases. Curr Pharm de-sign. 2014;20:652-8.
22. Martins Mdo C, Lima Faleiro L, Fonseca A. Relationship between leptin and body mass and metabolic syndrome in an adult population. Rev Port Cardiol. 2012;31:711-9.

23. Giulio Marchesini G. Lifestyle modification in the management of the metabolic syndrome: achievements and challenges. Diabetes, Metab Syndr Obes Targets Ther. 2010;373-385.

24. Julious SA. Sample size of 12 per group rule of thumb for a pilot study. Pharm Stat J Appl Stat Pharm Ind. 2005;4(4):287-91.

25. Keating XD, Zhou K, Liu X, Hodges M, Liu J, Guan J, et al. Reliability and concurrent validity of global physical activity questionnaire (GPAQ): a systematic review. Int J Environ Res Public Health. 2019;16(21):4128.

26. Jahangiry L, Shojaeizadeh D, Montazeri A, Najafi M, Mohammad $\mathrm{K}$, Yaseri M. Adherence, and attrition in a web-based lifestyle intervention for people with metabolic syndrome. Iran J Public Health. 2014;43(9):1248-58.

27. Rao DP, Orpana H, Krewski D. Physical activity and nonmovement behaviours: their independent and combined associations with metabolic syndrome. Int J Behav Nutr Phys Act. 2016;13(1):1-11.

28. King AC, Ahn DK, Oliveira BM, Atienza AA, Castro CM, Gardner CD. Promoting physical activity through hand-held computer technology. Am J Prev Med. 2008;34(2):138-42.

29. Marcus BH, Owen N, Forsyth LH, Cavill NA, Fridinger F. Physical activity interventions using mass media, print media, and information technology. Am J Prev Med. 1998;15(4):362-78.

30. Bice MR, Ball JW, McClaran S. Technology and physical activity motivation. Int J Sport Exerc Psychol. 2016;14(4):295-304.

31. Bort-Roig J, Gilson ND, Puig-Ribera A, Contreras RS, Trost SG. Measuring and influencing physical activity with smartphone technology: a systematic review. Sports Med. 2014;44(5):671-86.

32. Xenachis C, Samojlik E, Raghuwanshi MP, Kirschner MA. Leptin, insulin, and TNF-alpha in weight loss. J Endocrinol Invest. 2001;24:865-70.

33. Bastard JP, Jardel C, Bruckert E, Blondy P, Capeau J, Laville M, et al. Elevated levels of interleukin- 6 are reduced in serum and subcutaneous adipose tissue of obese women after weight loss. J Clin Endocrinol Metab. 2000;85:3338-42.

34 Afshin A, Babalola D, Mclean M, et al. Information technology and lifestyle: a systematic evaluation of internet and mobile interventions for improving diet, physical activity, obesity, tobacco, and alcohol use. J Am Heart Assoc. 2016;5(9):e003058.

35. Sampath Kumar A, Arun Maiya G, Shastry BA, Vaishali K, Maiya $\mathrm{S}$, Umakanth S. Correlation between basal metabolic rate, visceral fat, and insulin resistance among type 2 diabetes mellitus with peripheral neuropathy. Diabetes Metab Syndr. 2019;13(1):344-8.

36. Lien LF, Brown AJ, Ard JD, Loria C, Erlinger TP, Feldstein AC, et al. Effects of PREMIER lifestyle modifications on participants with and without the metabolic syndrome. Hypertension. 2007;50: 609-16.

37. Volek JS, Phinney SD, Forsythe CE, Quann EE, Wood RJ, Puglisi MJ, et al. Carbohydrate restriction has a more favourable impact on the metabolic syndrome than a low-fat diet. Lipids. 2009;44:297309.

38. Oh EG, Hyun SS, Kim SH, Bang SY, Chu SH, Jeon JY, et al. A randomized controlled trial of therapeutic lifestyle modification in rural women with metabolic syndrome: a pilot study. Metabolism. 2008;57:255-61.

Publisher's note Springer Nature remains neutral with regard to jurisdictional claims in published maps and institutional affiliations. 